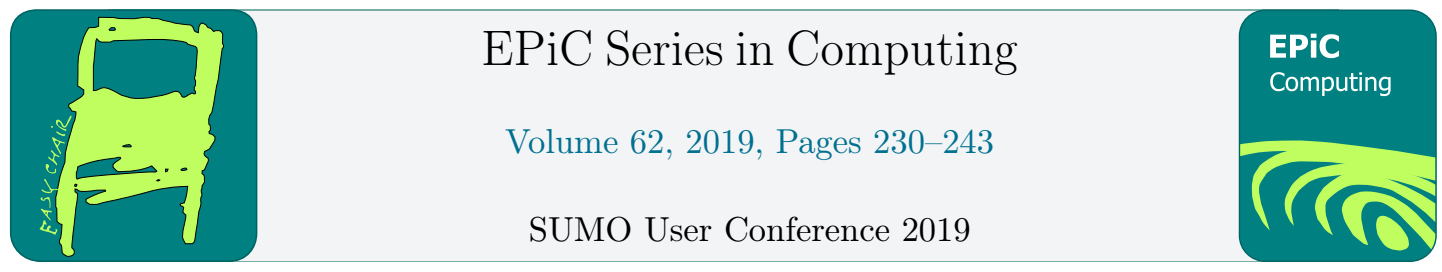

\title{
Anticipating Automated Vehicle Presence and the Effects on Interactions with Conventional Traffic and Infrastructure
}

\author{
Gerald Richter ${ }^{1,3}$, Lukas Grohmann ${ }^{1}$, Philippe Nitsche $^{2}$, and Gernot Lenz ${ }^{1}$ \\ 1 Dynamic Transportation Systems, Austrian Institute of Technology (AIT), Vienna, 1210, Austria \\ 2 Transportation Infrastructure Technologies, Austrian Institute of Technology (AIT), Vienna, 1210, \\ Austria \\ 3 gerald.richter@ait.ac.at
}

\begin{abstract}
Expectations are that automated and connected mobility will increase road safety and traffic efficiency. However, due to possible shortcomings of new technologies, road users may be confronted with disturbances and potential safety risks. The mitigation of such risks will bring necessary changes to road infrastructure, vehicles and road-users' behavior. In a traffic environment that was built to fit the human perception, preemptive simulation of parametrized scenarios can provide guidelines for what changes and difficulties are to be expected. Utilizing SUMO in varied scenarios, this paper outlines the creation of virtual models that correspond to interaction hot spots on the Austrian road network - from digitizing the infrastructure, to calibrating a simulation scenario with congruent traffic measurements - while it concludes with the evaluation of scenario simulation results. The approach is demonstrated for a selected motorway ramp scenario, varying rates of automated vehicles and different infrastructure layouts. Performance indicators like vehicle speed distributions and traffic disruptions are defined and analyzed to investigate how adaptations can mitigate risks, influence traffic flow and hence support progressing vehicle automation.
\end{abstract}

\section{Introduction}

The application of artificial intelligence is increasingly present in many parts of our lives. On the roads the capabilities and possible shortcomings of new technologies might cause disturbances and potential threats regarding what humans have come to perceive as a usual flow of traffic. An automated vehicle (AV) will, for instance, not be equipped with a comparable decision logic applied by a human driver who steers a conventional vehicle (CV). While the foreseeable developments in this field allow for a greatly improved safety in many traffic situations [13], this promise is uncertain to hold true for the period of mixed traffic with conventional road users.

Besides potential safety risks, this can lead to situations where traffic flow is easily interrupted by the strict adherence to high safety standards, causing an AV to stop or slow down at non-ideal conditions for a planned maneuver. Problematic factors that may cause such 
non-ideal conditions in traffic can be distinguished into three groups: (1) Road infrastructure characteristics and conditions such as limited distance of sight, poor surface or lane markings quality, (2) adverse weather conditions that lead to reduced sensor visibility and (3) misleading or insufficient interaction with other human road users.

The work conducted for this article was part of a larger Austrian research project called via-AUTONOM, which studied the requirements for future road infrastructure in the light of increased AV penetration. Simulation models and methods allow to preemptively study possible scenarios, at little cost and no risk of harm.

This publication demonstrates microscopic simulations in reconstructions of a select motorway on-ramp scenery to evaluate how an infrastructure adaptation influences the efficiency and safety of a mixed traffic flow. The software package for road traffic simulation SUMO [8] was chosen as the simulation tool due to its easy extensibility, good user support, as well as the transparency of an open-source project.

As indicators for the fluency of the traffic, we calculate the frequency of forced stops, the minimal time to collision (TTC), minimal vehicle spacing and the harmonization of the traffic flow after merging, which are detailed in section 3.3.

This research focuses on two key issues. First, it presents the accurate reconstruction of a road section from real-world measurements, and subsequent transfer to the OpenDRIVE format [4]. Second, a novel set of indicators was combined to derive findings about traffic efficiency in relation to an adapted road infrastructure. Ultimately, the methods presented form the basis to determine the optimal cost benefit trade-off for proposed infrastructure measures, by comparing the resulting impacts to expected costs for adapting or retrofitting current infrastructure.

This document is structured as follows: Section 2 presents the road layout and the traffic composition. Section 3 describes the data acquisition chain from road to virtual representation, the methods used for simulation and traffic flow evaluation. It is followed by a summary of results in section 4. The conclusions in section 5 and perspective on future work in section 6 conclude the report.

\section{Simulation Scenario}

The following section describes the required components that constitute the overall virtual representation of the investigated scenario, which comprise the setting's road topology and the traversing traffic.

\subsection{Road Layout}

A motorway segment with a ramp and an acceleration lane was chosen as the primary scenario for the presented research. The real-world location of this segment is near Exit 'Zöbern', on the Austrian motorway 'A2 - Süd Autobahn'. The speed limits on this motorway section is 100 $\mathrm{km} / \mathrm{h}$ for passenger cars.

Figure 1 shows the layout of the traffic situation. Cross-sectional speed detectors were introduced at different locations in the virtual representation of the scenery, represented by points 'C', 'P1' and 'P2'. Location $\mathrm{C}$ was used for measurements to calibrate a realistic behavior of the simulated traffic on the main lanes 0 and 1 (see section 3.2.2). Locations 'P1' and 'P2' were introduced to measure the differences in speed distributions at these points (see section 3.3).

Two different infrastructural settings were examined in this study. The first being the baseline situation of the real road infrastructure ("short" acceleration lane). An identical layout, but with an acceleration lane extended by $300 \mathrm{~m}$ was used as the second, modified infrastructural 
situation ("medium" acceleration lane). This was done to examine the effects on traffic flow, given an adapted infrastructure.

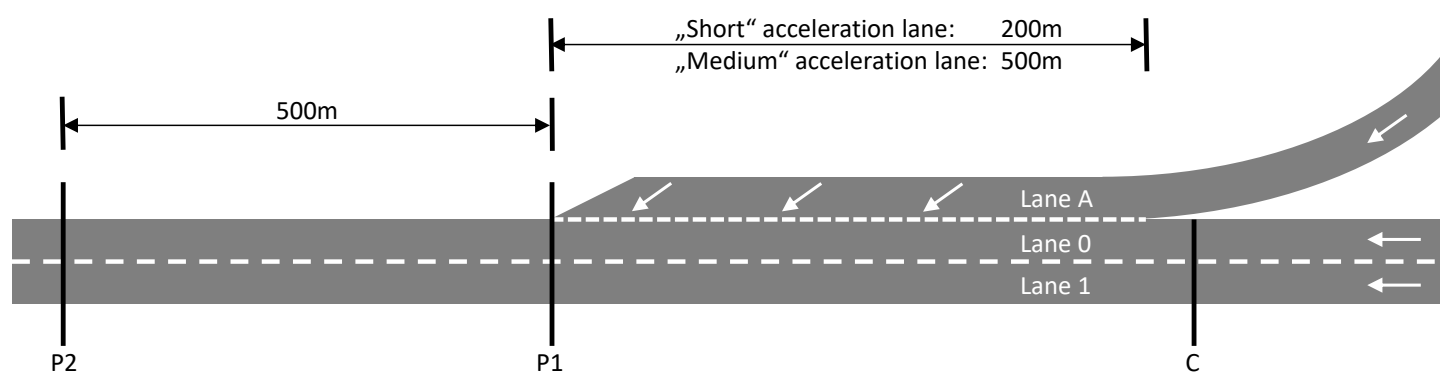

Figure 1: Scenario of traffic merging from right to left. Position $\mathrm{C}$ is the detector location for calibration, positions P1 and P2 are detectors for speed-profiling the consequences of merging. Lane A designates the acceleration lane with different lengths for the two infrastructural settings. The length of the acceleration lane is different for the settings of "short" and "medium" acceleration lane.

\subsection{Traffic Composition}

The traffic load at the scenario site was determined by using measurement data from roadside sensors that provided aggregated averages by vehicle category for a period of approximately 6 weeks. Segmentation of this data yields traffic counts of $(940 / 165)$ vehicles per hour for (passenger cars (PC) / heavy goods vehicles (HGV)) during the morning peak, and (625 / 170) vehicles during late morning, respectively. The higher load at morning peak hours was chosen as base value in the following investigations.

In the simulation model, the scenario traffic is composed of three different vehicle type compositions, one for each lane which are illustrated in Table $1.18 \%$ of total traffic on both lanes are HGV which are restricted to the right lane. This is kept constant for all considered traffic scenarios. Additionally, the composition contains two different types of CVs as well as AVs on all lanes, which uniformly make up all the PCs in the simulation. The mixed composition of CVs was chosen to better model the measured speed distributions of the scenario, thus improving the stability of calibration results (see section 3.2.2). Furthermore, to avoid an overly regular insertion of vehicles into the simulations, they were defined by a probability per second, resulting in a binomially distributed inflow.

\section{Methods}

Starting with the means to obtain the baseline data of the simulation scenarios, the choice of the simulation approach is presented in the following section. This includes the vehicle models, and is followed by scenario calibration and the description of indicators for traffic flow evaluation. 


\begin{tabular}{c||c|c|c}
$\begin{array}{c}\text { vehicle } \\
\text { type }\end{array}$ & lane 0 & lane 1 & lane A \\
\hline $\mathrm{CV} 1$ & + & 0 & + \\
$\mathrm{CV} \mathrm{2}$ & 0 & + & + \\
$\mathrm{HGV}$ & $\approx 18 \%$ tot. traffic & 0 & 0 \\
$\mathrm{AV}$ & $0-70 \% \mathrm{CV}$ & $0-70 \% \mathrm{CV}$ & $0-70 \% \mathrm{CV}$
\end{tabular}

Table 1: Traffic inflow composition given by vehicle type components per lane (given in \%). '+' indicates presence of inflow of different volumes, depending on the scenario setting. See Figure 1 for road layout.

\subsection{Digitalization of Roads}

A tool chain was developed to digitalize real-world road sections for simulation purposes. It consists of four steps, which are depicted in Figure 2.

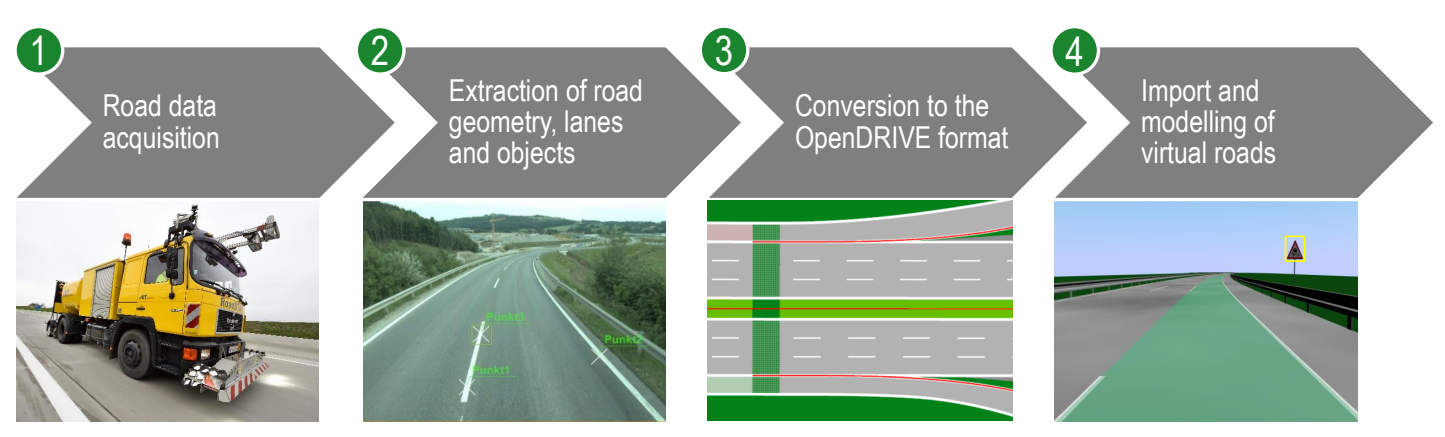

Figure 2: Tool chain to reconstruct real-world roads for simulations.

As the simulations are done on reconstructed real-world road sections, the modelling is based on measurements conducted by a dedicated mobile laboratory called RoadSTAR [16]. The road data consists of stereo-video streams recorded during a large-scale road condition monitoring campaign on the Austrian motorway network. Additionally, a precise trajectory was generated from positioning and attitude data from a tightly-coupled IMU and GNSS system. An in-house mobile mapping work flow was utilized to manually extract road characteristics such as lane width, lane markings and roadside objects, e.g. traffic signs or restraint systems. The extracted objects and their coordinates were stored in a database and mapped to a global coordinate system. A software was developed to convert the object database to the XML-based OpenDRIVE format [4], which allows a standardized and detailed description for wider application in the future.

To generate a simulation network based on the real-world data, the SUMO tool NETCONVERT was used for converting the data from OpenDRIVE format to the likewise XML-based SUMOnetwork format [8]. Subsequently, a validation of the obtained SUMO network ensured the two network representations to coincide. A prohibition rule for trucks and trailers was added to the left main motorway lane of the SUMO-network, which effects the situation of prohibited overtaking for HGVs. 


\subsection{Simulation}

The investigation of traffic flows containing a multitude of AVs is the aim of this study. To simulate the behavior of automated vehicles, different alternatives to model AVs in the SUMO environment were considered. A co-simulation approach for a simulated AV requires a well defined interface that transforms and exchanges all information between vehicle and environment. For the simulation of many AVs this most versatile method soon becomes computationally prohibitive. Therefore, a strategy for modeling was chosen that has been presented as a viable solution in other traffic micro simulation studies utilizing SUMO [11, 18], as well as other simulation tools [9]. The parameters of SUMO's standard car following model were adapted to best mimic the behavior of automated vehicles. This is technologically identical to a conventional simulated vehicle model while it offers all the benefits of efficiency and stability.

\subsubsection{Vehicle Model}

Due to its simplicity and reliability, a modified version of the model Krauss was chosen for modeling the vehicle behavior. This microscopic, space-continuous car following model based on the safe speed (according to [10]) is documented as the default model of SUMO in [2]. In contrast to other car following models (CFM), e.g. the Wiedemann model used in VISSIM [5], it only consists of a small set of significant input parameters, which have to be adapted appropriately for describing AVs. The set of parameters used in the simulations within this project are shown in Table 2 and were chosen based on the following assumptions:

All AVs aim at driving with their desired speed, which is equal to the maximum speed limits in the simulation scenarios. Therefore, the variance of the speed distribution was set to zero in order to avoid speed deviations, which is one of the important differences compared to CVs. In addition, the drivers imperfection described by sigma was set to zero (compare [1]). Furthermore, reaction times of AVs are defined by technological limitations and therefore can be shorter than those of human drivers [19]. To account for this the actionStepLength (aSL), which describes the periodicity of vehicle-control decision updates of the CFM, was set to smaller values for AVs. As a consequence, the desired minimum time headway tau of AVs was reduced, allowing a denser traffic flow. Consequential effects are discussed in section 4 .

In this study, identical acceleration values for $\mathrm{AVs}$ and $\mathrm{CVs}$ were set due to the focus on delicate merging situations and emergency maneuvers. This is in contrast to other studies, which also consider the more energy-efficient driving behavior of AVs and assume reduced values for these parameters. However, in the presented investigations, such efficiency limitations should not be applied. In general, AV parameters were set compliant with SAE level 5 in [15]. However, for the purposes of this study, vehicle connectivity and its effects on driving decisions were disregarded.

For the simulations, the continuous lane-change model of SUMO was chosen in order to represent the more realistic aspects of non-instantaneous lane-changing as suggested in [2]. Some

adaptions of the lane-change model parameters were made to describe the assumed behavior of AVs. In essence (see Table 2), more strategic and cooperative lane changing behavior of AVs in comparison to CVs was effected.

\subsubsection{Calibration}

Actual roadside detector data (see section 2.2) was used to compare against data from the simulation's instantaneous induction loops (IIL) taken at cross section 'C' in Figure 1. Preparation of the IIL data enables to treat the simulated detectors identical to the real ones. 


\begin{tabular}{c||c|c|c|c|c|c|c|c}
$\begin{array}{c}\text { vehicle } \\
\text { type }\end{array}$ & accel & decel & tau* & sigma & aSL & speedFactor* & lcS & lcC \\
\hline CV 1 & 2.0 & 4.0 & 1.07 & 0.5 & 0.7 & $\mu=1.03, \sigma=0.103$ & 0.8 & 0.5 \\
CV 2 & 2.0 & 4.0 & 1.07 & 0.5 & 0.7 & $\mu=1.04, \sigma=0.161$ & 0.8 & 0.5 \\
HGV & 2.0 & 4.0 & 1.21 & 0.5 & 0.7 & $\mu=0.91, \sigma=0.115$ & 1.0 & 0.5 \\
\hline AV & 2.0 & 4.0 & 0.4 & 0.0 & 0.1 & $\mu=1.00, \sigma=0.000$ & 5.0 & 1.0
\end{tabular}

Table 2: Car-following and lane-changing Parameters for both classes of CV and HGV for the Krauss car-following model. Optimized parameters are shown as starred* and are a result of the the calibration. Using the same model for $\mathrm{AV}$, the last line shows the chosen parameters. In this context, aSL denotes the actionStepLength, lcS denotes the parameter lcStrategic and $1 \mathrm{cC}$ denotes the parameter lcCooperative.

For the calibration of the baseline scenario the parameters of the desired speed normal distributions were optimized separately for both CV types and the HGV type. An additional minimum time headway parameter was used for the $\mathrm{CV}$ and $\mathrm{HGV}$, resulting in a total of eight parameters $(\{\mu, \sigma\} \times\{\mathrm{CV} 1, \mathrm{CV} 2, \mathrm{HGV}\}$ and $\{\tau\} \times\{\mathrm{CV}, \mathrm{HGV}\})$ for optimization. The distribution splitting of the $\mathrm{CV}$ vehicle class allows for a wider speed distribution in the measured data, which thereby is expressed as a gaussian mixture of two components. Using the Manhattan distances of speed distribution probability mass functions (PMF) weighted by the counts of the detected vehicle class $(\mathrm{CV}, \mathrm{HGV})$, provides a sufficient optimization criterion for the given scenario. To check the stability of obtained optimization solutions, two different optimization algorithms (swarm optimization [17] and a bayesian optimization [7]) were used which both yielded similar numerical results.

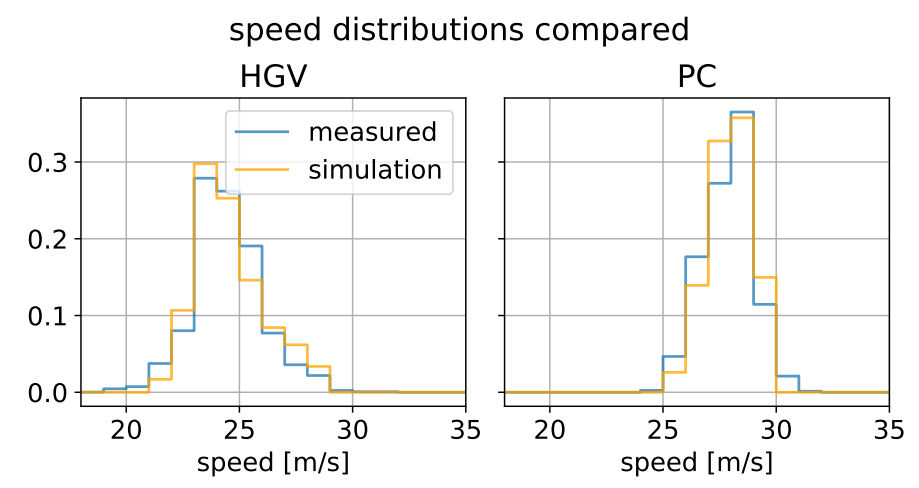

Figure 3: Compared distributions of vehicle speeds by types heavy goods vehicle (HGV), passenger car (PC) for roadside measurements and simulation detector outputs of calibrated scenario. The optimization yields good similarity of the speed profiles.

The optimization results in Figure 3 show the desired speed distributions, comparing the real measured PMF for both vehicle types currently encountered on the scenarios' roads (CV, HGV) against the simulated measurements. 


\subsubsection{Scenario Variations}

Considering possible settings for the chosen scenario location led to the choice of three different traffic composition parameters that are described in Table 3. The cartesian product of all three sets leads to 120 different scenario variations, to be simulated and analyzed. To derive solid statistical conclusions on the effects of the scenario variations, each of the parameter settings was run in repeated simulations for a total of 100 hours each.

\begin{tabular}{|c|c|}
\hline main traffic scale & $1.0,1.5,2.0,2.5$ \\
\hline AV penetration rate & $0,0.1,0.2,0.3,0.5,0.7$ \\
\hline inflow rate & $0.1,0.2,0.4,0.6,0.8$ \\
\hline
\end{tabular}

Table 3: Sets for each considered scenario parameter. The inflow rate yields the traffic on lane A in relation to lane 0 (compare Figure 1).

\subsection{Indicators for Safety and Traffic Flow}

Considering the safety conditions and fluency of the traffic under scenario variations, the following indicators were implemented:

- Frequency of severe interruptions When AVs react to situations that can not be handled safely by their steering functions they will be forced to halt the vehicle. This also affects other oncoming traffic. This aspect was implemented by analyzing the number of forced stops on the acceleration lane A.

- Harmonization of traffic after merging Because freeway flow disturbances and oscillations often form near ramps where traffic merging happens (as shown in [12]), it is of special interest to monitor the restored uniformity of traffic after the end of the acceleration lane. The speed distributions at points 'P1' and 'P2' in Figure 1 are utilized as a measure to characterize and compare flow uniformity by speed mean values and variances.

- Vehicle spacing

A popular temporal indicator to distinguish critical from normal driving behavior is the Time to Collision (TTC) [6], which is used for traffic conflict analysis and collision avoidance systems as a measure for the imminence of a collision. Complementary to the TTC, spatial distance provides another perspective on potential conflicts. Occurrence of close vehicle proximity results in challenging driving situations.

\section{Results}

In the following, results will be presented for the three defined indicator aspects.

\subsection{Flow Interruptions}

Flow interruptions are caused by the rush of merging traffic. As the first indicator of trafficfluency, the number of forced stops of vehicles on the acceleration lane is investigated. A normalization to the inflow traffic volume was carried out to equalize the following results. 


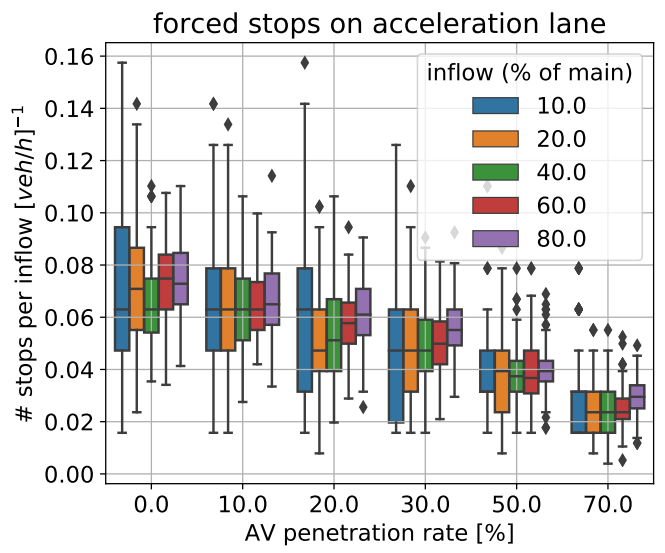

Figure 4: Box plot of forced stops on 'lane A' normalized to inflow for $100 \%$ main traffic scaling and "short" acceleration lane.

For the baseline scenario ("short" acceleration lane) and nominal traffic flow on the main lanes (main lanes' traffic at $100 \%$ ), Figure 4 shows a reduction of stop occurrences per inflow strength with increasing penetration of AVs. The same scenario with $250 \%$ main lane traffic load in Figure 5a, exhibits a drastic increase of the median values at a factor of $\geq 2.5$ in comparison to Figure 4. Increasing inflow leads to excessively more vehicle stops - especially for low AV penetration rates. Higher AV rates result in a number of stops less sensitive to the inflow. The cause for this effect is two-fold. On the one hand, AVs exhibit a more conservative driving style, with homogeneous speed choice, higher cooperativity and no driving errors. On the other hand, a greater traffic volume leads to more tightly coupled vehicle behavior, where $\mathrm{AVs}$ enforce their style onto others.

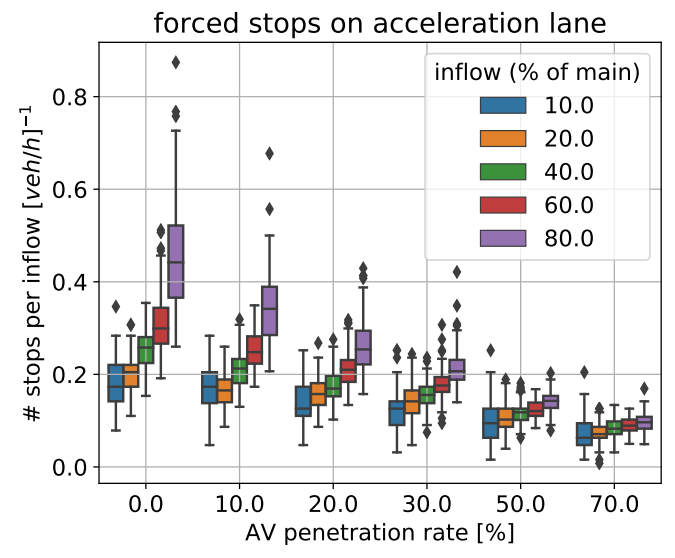

(a) "short" acceleration lane.

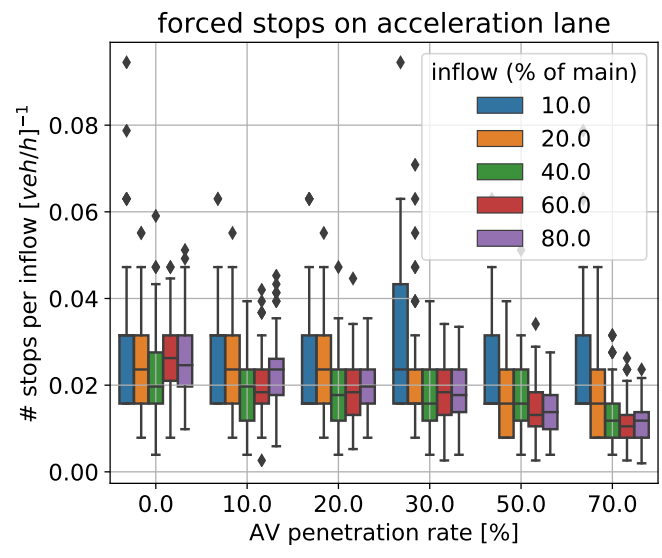

(b) "medium" acceleration lane.

Figure 5: Box plot of forced stops on 'lane A' normalized to inflow for $250 \%$ main traffic scaling. 
With modified road infrastructure ("medium" acceleration lane) a very low number of stops can be seen. Figure $5 \mathrm{~b}$ shows median values of approximately $1 / 10$ of those for the same traffic scaling in the baseline with "short" acceleration lane (Figure 5a). The long distance allows for enough time to maintain fluent traffic. Yet the effects of increased AV rates are discernible.

\subsection{Traffic Homogeneity}

As a measure for the homogenization of downstream-traffic after the end of the acceleration lane, two detectors for cross-sectional speed measurements were introduced in the simulation. In the diagram of Figure 1, their locations are 'P1' and 'P2'. The space between those points is considered as traffic 'adaptation zone'. Aggregation of these detector measurements into speed distribution PMFs (similar to those used for calibration in Figure 3) allows inspection and comparison of the traffic in both detection points. The following analysis shows the results for passenger cars only.

For the sake of presentation as well as comparability, the considerable amount of information contained in those measurements for all parameter settings is reduced to weighted averages (average) and standard deviations (std) of the measured speed. Because the changes of the speed distributions between the two detection points is of major interest, relative differences $r\left(P_{1}, P_{2}\right)$ of average and std are calculated as $r\left(P_{1}, P_{2}\right)=\left(v\left(P_{2}\right)-v\left(P_{1}\right)\right) / v\left(P_{1}\right)$ with $v$ being the value of either average or std at the respective detection points (i.e.: how much do those values change in relation to the value at $P_{1}$ ). These values are computed for all cases of defined $v\left(P_{2}\right)$ and $v\left(P_{1}\right)$.

The baseline scenario ("short" acceleration lane) and nominal traffic flow on the main lanes (main lanes' traffic at $100 \%$ ) is summarized in Figure 6. Reading this diagram reveals the following: For high inflow on lane A (80\%) and low AV penetration rate $(0 \%)$, the relative average speed (left green diagram) increases by about 35\% throughout the adaptation zone and has a starting value of $21 \mathrm{~m} / \mathrm{s}$ at point 'P1'. The relative standard deviation of the speeds (right blue diagram) for the same inflow and AV penetration reduces by $80 \%$ throughout the adaptation zone, with a start value of $5 \mathrm{~m} / \mathrm{s}$ at point 'P1', effectively reducing the spread of PC speeds significantly.

When considering high main traffic load of $250 \%$ for the "short" acceleration lane setting, the consequences are shown in Figure 7. High inflow and low AV penetration significantly reduce the average speeds right after the acceleration lane in point 'P1'. More AVs cause significantly less of these speed reductions due to merging. The right std diagram shows little spread (4.1) for high inflow and no AVs, which indicates coupled vehicle behavior. An increasing AV rate leads to better speed synchronization and higher average speeds.

Comparing against the case of high traffic load of $250 \%$ on the medium acceleration lane scenarios shows that relative speed increases are smaller, because the traffic flow is almost undisturbed after the acceleration lane. The increasing the number of AVs causes higher uniformity of speeds.

\subsection{Vehicle Spacing}

To investigate the safety impact of AVs, the safety surrogate measurements (SSM) of the implemented simulation scenarios are analyzed. All simulated passenger cars (CV1, CV2, AV) to that effect were equipped with an SSM device, which logs the conflicts and corresponding safety surrogate measures. With our focus set on passenger cars, no measurements were recorded for the unmodified HGV traffic. Furthermore, this section only includes results for the scenario with short acceleration lane and a main traffic scaling of $250 \%$. The demonstrated effects are 


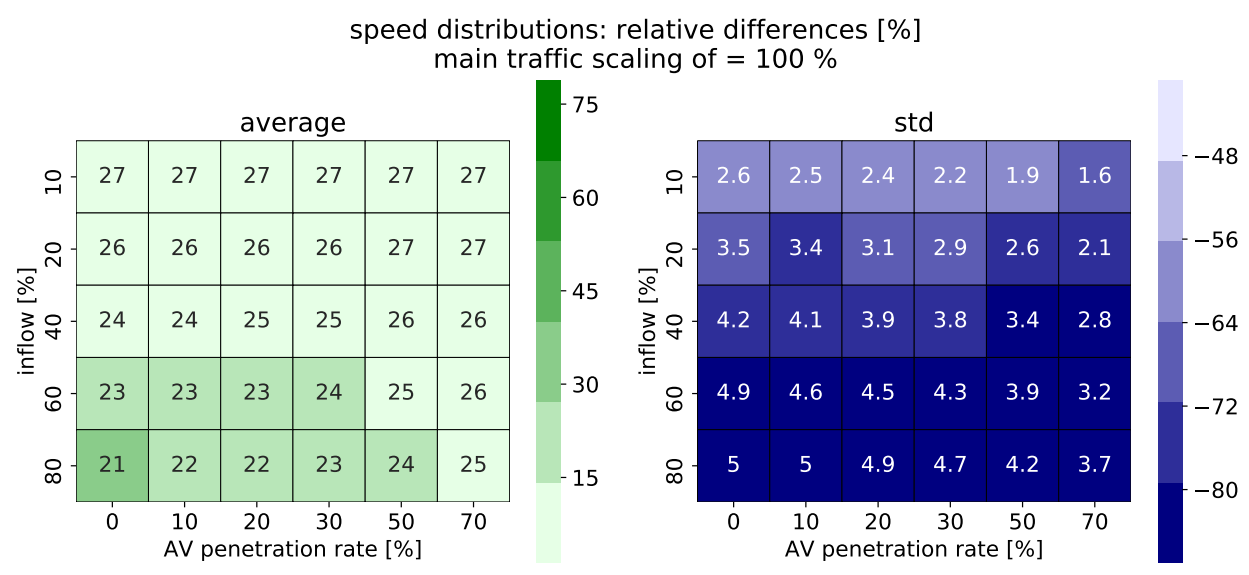

Figure 6: Relative change of weighted average and standard deviation of PC speeds between the detection points for 100\% main traffic scaling and "short" acceleration lane. Color indicates the relative change in \%. Absolute values at detector 'P1' are annotated as $[\mathrm{m} / \mathrm{s}]$ in the cells.

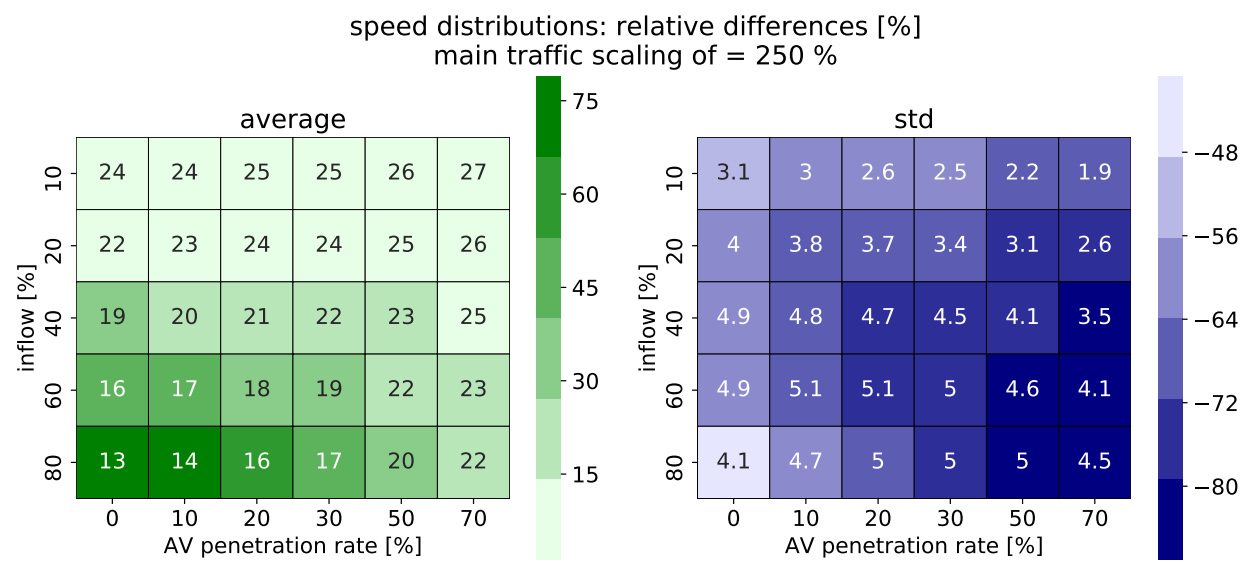

Figure 7: Relative change of weighted average and standard deviation of PC speeds between the detection points for $250 \%$ main traffic scaling and "short" acceleration lane. Color indicates the relative change in \%. Absolute values at detector 'P1' are annotated as $[\mathrm{m} / \mathrm{s}]$ in the cells.

more pronounced for this most critical setup, where the available time for completion of the merging maneuvers is limited.

For estimating the number of occurrences of critical situations, it is generally assumed that whenever a vehicle's TTC falls below a certain value, this can be considered as an unsafe situation [3]. Based on previous research on the safety impact of AVs [14], the critical threshold value was set to 1.5 seconds. In addition to the investigation of the number of conflicts, the minimal time and space headway were analyzed. 
In Figure 8, the impact of the AV penetration rate on the number of conflicts per simulation hour (left) and mean values of the average minimal spacings (right) for different inflows are shown. The abolute number of conflicts considerably reduces even for high inflows, which is in accordance with the results obtained in [14]. Here, both the number of conflicts and minimal spacing tend to converge against a specific value defined by the increasing predominance of automated vehicles.
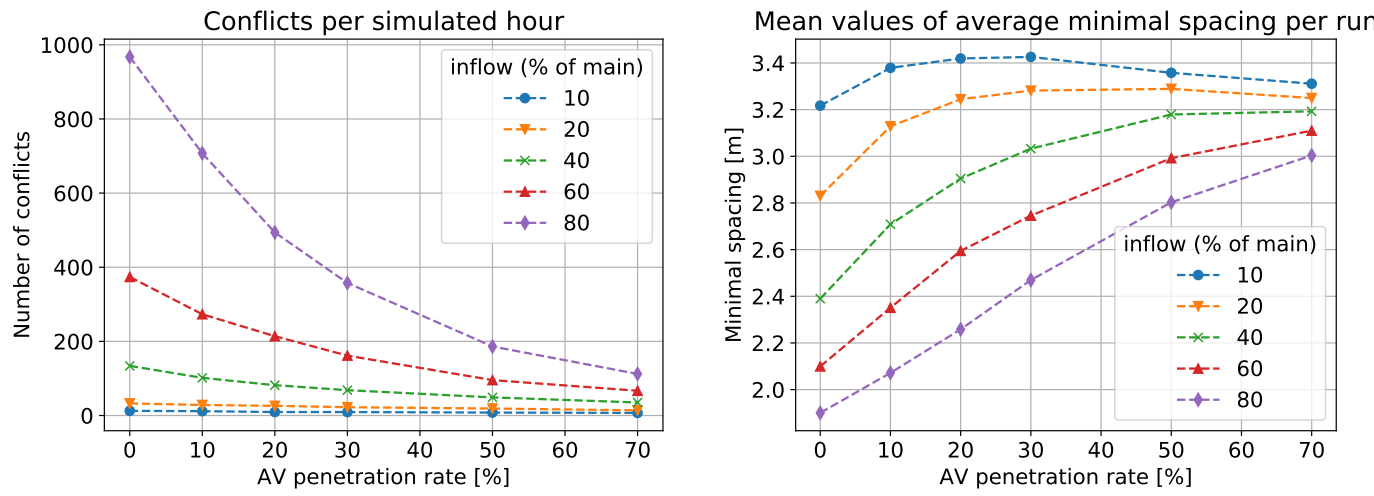

Figure 8: Impact of the AV penetration rate on the absolute number of conflicts (TTC $\leq 1.5$ seconds) occuring in one simulation hour (left) and the mean values of all run average minimal spacings (right) for different traffic inflows. Data point errors are $\leq 1 \%$. The figures show a main traffic scaling of $250 \%$ with short acceleration lane.

This convergence behavior at increasing AV penetration shows the mean value of the minimal spacings to increase (Figure 8 right), due to less critical situations occurring by the moderating influence of AVs. With further increase of AV penetration, for low inflows, the smaller headway distances of AVs reduce the minimal spacings still, while numbers of conflicts decrease.

\section{Conclusions}

This research studied the infrastructural modification of an on-ramp motorway section under different traffic compositions, especially varying autonomous vehicle penetration rates. To this end multiple traffic scenarios were simulated for each of two different infrastructural settings.

Implementing the infrastructure measure of a significant extension of the acceleration lane for the studied motorway ramp scenario, strongly reduces the effects of interruptions arising due to limited opportunities for merging into the main lanes' traffic.

Similar mitigation effects can be achieved with an increasing penetration rate of automated vehicles, partially replacing the passenger cars as conventional vehicles. AV penetration rates of $15-25 \%$ already show considerable improvements regarding the investigated indicators of forced stops, traffic harmonization (uniformity) and the safety surrogate measures of TTC and minimal space gap. As a consequence, the smaller minimum time headway of AVs coincide with a reduced number of conflicts that are expressed by the emergence of situations with a TTC below threshold.

Conclusively, this research demonstrates that properly scaled adaptions of the road infrastructure can ease the transition towards increasing automation of vehicles. With an extension 
of the methods presented here, traffic micro-simulations allow to better determine the optimal extent of such adaptions. This can also be applied inversely to locate the spots within a road network that may require infrastructural adaptations. If constructional measures such as the extension of lanes are infeasible, infrastructure-to-vehicle digital systems can be implemented.

The presented approach can therefore be extended to support infrastructure operators in their decisions to prepare for the rise of automated mobility.

\section{Outlook}

With the outlined strategy of this research, several key issues have to be addressed in the future to help further improve upon results that can be derived by the presented approach.

To allow for a good prediction of the traffic situation at a given site of interest, detailed traffic data is required. This includes estimates of expectable penetration rates of AVs. Improvement upon the vehicle model of AVs by implementing one of the other presented options allows for even more realism and predictability. Future work will certainly address the point of allowing for a parametrized change of road topology, e.g. changing the length of the acceleration lane in our presented example by a 'free' parameter and optimizing it for any given traffic situation. The inclusion of different vehicle automation levels as well as vehicle communication capabilities show great promise for more detailed investigations.

Regarding the extent of infrastructure measures to prepare for vehicle automation, it becomes important to estimate a cost / benefit balance for road adaptions using road construction or stationary electronic means to provide the required traffic safety and fluency, depending on technical capabilities of vehicles.

\section{Acknowledgements}

The project "Verkehrsinfrastruktur und Anforderungen für autonomen Straßenverkehr" (viaAUTONOM) was funded by the Austrian Federal Ministry for Transport, Innovation and Technology through the grant program "Mobilität der Zukunft" (6th call 2015, grant number 854991). We would like to thank the ASFINAG Maut Service GmbH for providing traffic count data at the investigated motorway segment at Exit 'Zöbern', on the Austrian motorway 'A2 - Süd Autobahn'. Also, we acknowledge the contributions from our project partners: "Kompetenzzentrum - Das virtuelle Fahrzeug, Forschungsgesellschaft mbH", "PRISMA solutions EDV-Dienstleistungen GmbH", "Rosinak\&Partner ZT GmbH", "Wieser Verkehrssicherheit GmbH"

\section{Author Contributions Statement}

G.R. original draft preparation; G.R. and L.G. methodology, simulations, software and validation; G.R. and L.G. carried out data analysis and result visualization; G.R., P.N. and L.G. wrote the paper; G.L. proof-reading and visualization; P.N. project conceptualization, administration, funding acquisition, review. All authors reviewed the manuscript. 


\section{References}

[1] Erik Bjärkvik, Florian Fürer, Mitra Pourabdollah, and Björn Lindenberg. Simulation and Characterisation of Traffic on Drive Me Route around Gothenburg using SUMO. In Proceedings of the SUMO2017 - Towards Simulation for Autonomous Mobility, pages 1-13. Deutsches Zentrum für Luft- und Raumfahrt e. V., Institut für Verkehrssystemtechnik, 2017.

[2] SUMO developer community. Car-following models (online documentation). https://sumo.dlr. de/wiki/Definition_of_Vehicles,_Vehicle_Types,_and_Routes\#Car-Following_Models. Accessed: 2019-02-26.

[3] Atze Dijkstra, Paula Marchesini, Frits Bijleveld, Vincent Kars, Hans Drolenga, and M.F.A.M. Maarseveen. Do calculated conflicts in microsimulation model predict number of crashes? Transportation Research Record Journal of the Transportation Research Board, 2147:105 - 112, 122010.

[4] Marius Dupuis. OpenDRIVE - Format Specifications, Rev. 1.4. OpenDRIVE Document VI2014.106, VIRES Simulationstechnologie GmbH, November 2015.

[5] Martin Fellendorf and Peter Vortisch. Microscopic Traffic Flow Simulator VISSIM, pages 63-93. Springer, New York, New York, NY, 2010.

[6] J. C Hayward. Near-miss determination through use of scale of danger. Highway Research Record, 384:24-34, 1972. OCLC: 173427170.

[7] Tim Head, Gilles Louppe, Iaroslav Shcherbatyi, et al. skopt module. https://github.com/ scikit-optimize/scikit-optimize, 2016-2019.

[8] Daniel Krajzewicz, Jakob Erdmann, Michael Behrisch, and Laura Bieker-Walz. Recent Development and Applications of SUMO - Simulation of Urban MObility. International Journal On Advances in Systems and Measurements, 3\&4, 122012.

[9] Sabine Krause, Nassim Motamedidehkordi, Silja Hoffmann, and Fritz Busch. Mikroskopische Simulation von automatisierten Fahrzeugen zur Ermittlung der Wirkungen auf die Kapazität von Autobahnen. Straßenverkehrstechnik, 2017.

[10] S Krauß. Microscopic modeling of traffic flow: Investigation of collision free vehicle dynamics. DLR - Forschungsberichte, 1998.

[11] Qiong Lu and Tamas Tettamanti. Impacts of autonomous vehicles on the urban fundamental diagram. In Road and Rail Infrastructure V, pages 1265-1271, 2018.

[12] Michael Mauch and Michael J. Cassidy. Freeway Traffic Oscillations: Observations And Predictions, pages 653-673. Emerald Group Publishing Limited, jun 2002.

[13] Dimitris Milakis, B Arem, and Bert van Wee. Policy and society related implications of automated driving: A review of literature and directions for future research. Journal of Intelligent Transportation Systems Technology Planning and Operations, 21:324-348, 022017.

[14] Mark Mario Morando, Qingyun Tian, Long T Truong, and Hai L Vu. Studying the Safety Impact of Autonomous Vehicles Using Simulation-Based Surrogate Safety Measures. Journal of Advanced Transportation, 2018:1-11, 2018.

[15] SAE. Taxonomy and Definitions for Terms Related to Driving Automation Systems for On-Road Motor Vehicles (J3016). Technical report, Society for Automotive Engineering, 2016.

[16] Roland Spielhofer and Torsten Schönberg. Präzise Verortung von Straßeninfrastruktur als Grundlage für eGovernmentprozesse. In Angewandte Geoinformatik 2010. Beiträge zum 22. AGITSymposium Salzburg, pages 426-434, 2010.

[17] tisimst@gmail.com. Particle Swarm Optimization (PSO) with constraint support. https: //pythonhosted.org/pyswarm, 2014-2015.

[18] François Vaudrin, Jakob Erdmann, and Laurence Capus. Impact of Autonomous Vehicles in an Urban Environment Controlled by Static Traffic Lights System. In Proceedings of the SUMO201\%. Deutsches Zentrum für Luft- und Raumfahrt e. V., Institut für Verkehrssystemtechnik, 2017.

[19] Yuguang Wei, Cafer Avcı, Jiangtao Liu, Baloka Belezamo, Nizamettin Aydin, Pengfei Li, and Xuesong Zhou. Dynamic programming-based multi-vehicle longitudinal trajectory optimization 
with simplified car following models. Transportation Research Part B: Methodological, 106:102-129, 122017. 